\title{
Structural mechanism of VWF D'D3 dimer formation
}

Zimei Shu ${ }^{1}$, Jianwei Zeng ${ }^{2,3 凶}$, Li Xia $^{1}$, Haiyan Cai ${ }^{1 凶}$ and Aiwu Zhou ${ }^{1 凶}$

Dear Editor,

Von Willebrand factor (VWF) assembly begins in the endoplasmic reticulum of endothelial cells and megakaryocytes where VWF is synthesized as a precursor with multiple domains (Fig. 1a). The polypeptide including the propeptide and mature VWF chain (proVWF) subsequently forms "tail-to-tail" homodimers through their C-terminal cystine knot (CK) domains. These proVWF dimers are then transported to the Golgi where they assemble into large multimers 'head-to-head' through interchain disulfide bonds between D3 domains of two proVWF dimers ${ }^{1-3}$. The Sadler group firstly identified a peptide containing the Cys ${ }^{1142}-\mathrm{Cys}^{1142^{\prime}}$ disulfide bond from plasma multimeric $\mathrm{VWF}^{4}$. Subsequently, through differential alkylation, proteolytic digestion, and mass spectrometry, they identified two free cysteines Cys ${ }^{1099}$ and $C y s^{1142}$ from $D^{\prime} D 3$ monomer and proposed their involvement in $\mathrm{D}^{\prime} \mathrm{D} 3$ dimer formation ${ }^{5}$. However, the crystal structure of a monomeric D'D3 mutant (C1099A/ C1142A) reported by Dong and Springer revealed that residue $\mathrm{Cys}^{1099}$ is largely buried inside of the $\mathrm{D}^{\prime} \mathrm{D} 3$ domain and it has to undergo a dramatic conformational change to allow forming a disulfide bond with the same cysteine from the other $\mathrm{D}^{\prime} \mathrm{D} 3$ molecule $^{6,7}$. Furthermore, the structures of dimeric D3 domains of Mucin 2 (MUC2), a homologous multimeric protein of VWF, showed Cys ${ }^{1142}-\mathrm{Cys}^{1142^{\prime}}$ and $\mathrm{Cys}^{1097}-\mathrm{Cys}^{1097^{\prime}}$ disulfide linkages (using VWF residue numbering) ${ }^{8}$. Cys ${ }^{1091}$

Correspondence: Jianwei Zeng (ceng-jw13@tsinghua.org.cn) or Haiyan Cai (hycai@shsmu.edu.cn) or Aiwu Zhou (awz20@shsmu.edu.cn) ${ }^{1}$ Department of Pathophysiology, Key Laboratory of Cell Differentiation and Apoptosis of Chinese Ministry of Education, Shanghai Jiao Tong University School of Medicine, Shanghai, China

${ }^{2}$ State Key Laboratory of Membrane Biology, Beijing Advanced Innovation Center for Structural Biology, School of Life Sciences, Tsinghua University, Beijing, China

Full list of author information is available at the end of the article

These authors contributed equally: Zimei Shu, Jianwei Zeng, Li Xia formed an intramolecular disulfide bond with $\mathrm{Cys}^{1099}$ in MUC2 instead of the Cys ${ }^{1091}-\mathrm{Cys}^{1097}$ seen in the crystal structure of the VWF D'D3 mutant. Therefore, the disulfide linkages and the cysteines involved in the VWF D'D3 dimer interface remain to be confirmed with the discrepancy mainly on Cys ${ }^{1097}$ and Cys ${ }^{1099}$ (Fig. 1a). Here we identified the disulfide linkages from the $\mathrm{D}^{\prime} \mathrm{D} 3$ dimer interface through chemical digestion and mass spectrometry and solved the cryo-electron microscopy (cryo-EM) structure of VWF tubules with each repeating unit containing one $\mathrm{D}^{\prime} \mathrm{D} 3$ dimer and one D1D2 dimer.

As the disulfide linkage of $\mathrm{Cys}^{1099}$ or $\mathrm{Cys}^{1097}$ from the VWF D'D3 dimer interface has never been identified and no suitable enzymic cleavage sites near the dimeric interface could be utilized to distinguish the cysteines of our interest, namely Cys ${ }^{1091}$, Cys ${ }^{1097}$, and Cys ${ }^{1099}$, here we designed a series of $\mathrm{D}^{\prime} \mathrm{D} 3$ mutants with residues flanking Cys ${ }^{1097}$, $\mathrm{Cys}^{1099}$, or $\mathrm{Cys}^{1142}$ mutated to methionines (Fig. 1b), and identified the intermolecular disulfide linkages directly by $\mathrm{CNBr}$ cleavage and mass spectrometry. For the D'D3 dimer variant with $\mathrm{Glu}^{1092}$ and $\mathrm{Ala}^{1098}$ flanking $\mathrm{Cys}^{1097}$ mutated to methionines (D'D3-E1092M-A1098M), a doubly charged parent ion with $m / z=576.223$ corresponding to the predicted Cys ${ }^{1097}$-Cys ${ }^{1097^{\prime}}$ disulfide-linked peptide $\left({ }^{1093}\right.$ SIGDC $\left.(\mathrm{Hsl})^{1098}\right)_{2}$ where $\mathrm{M}$ was converted to homoserine lactone (Hsl) could be readily identified. The identity of this peptide was further confirmed by the MS/MS spectrum of this ion with all the expected ions of peptide fragments detected such as $\mathrm{y} 4(\mathrm{~m} / \mathrm{z}=951.321, \mathrm{z}=1)$ and $\mathrm{b} 2(\mathrm{~m} / \mathrm{z}=201.124$, $\mathrm{z}=1$ ) (Fig. 1b). Similarly, a doubly charged ion with $m / z=796.270$ was identified from the dimer of the $\mathrm{D}^{\prime} \mathrm{D} 3$ variant (D'D3-R1136M-E1143M), which corresponded to the $\mathrm{Cys}^{1142}-\mathrm{Cys}^{1142^{\prime}}$ disulfide-linked peptide $\left({ }^{1137}\right.$ ENGYEC $\left.(\mathrm{Hsl})^{1143}\right)_{2}$ (Supplementary Fig. S1a). Notably, the disulfidelinked peptides where one or two methionines were converted to homoserines could also be readily identified (Supplementary Fig. S1b, c). Therefore, these results 
$\mathbf{a}$

SP $\frac{\text { Propeptide Furin }}{\text { D1 }}$

b

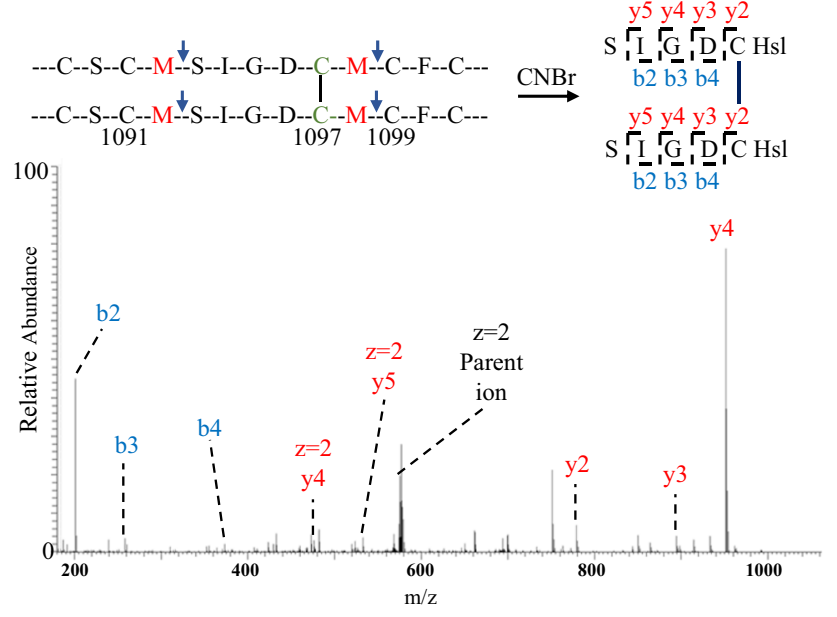

c

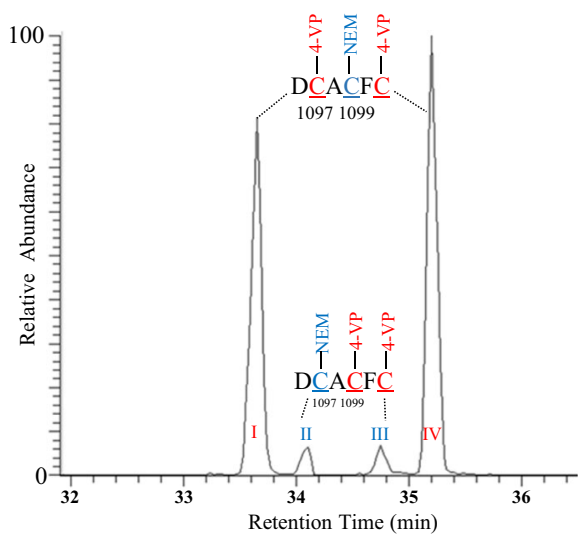

f

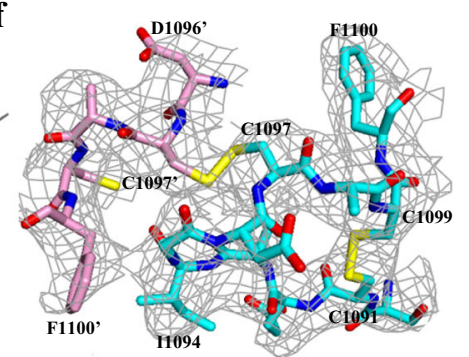

g
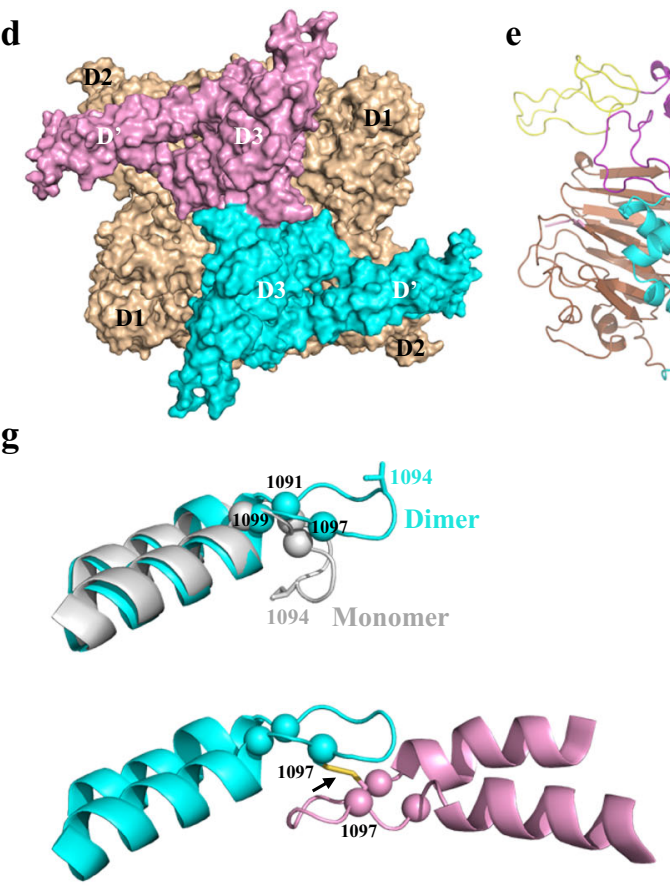

D'D3 dimer

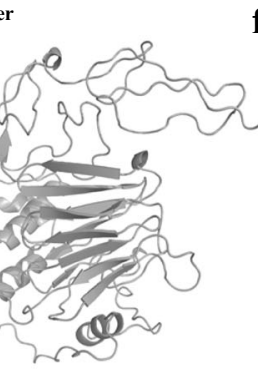

h
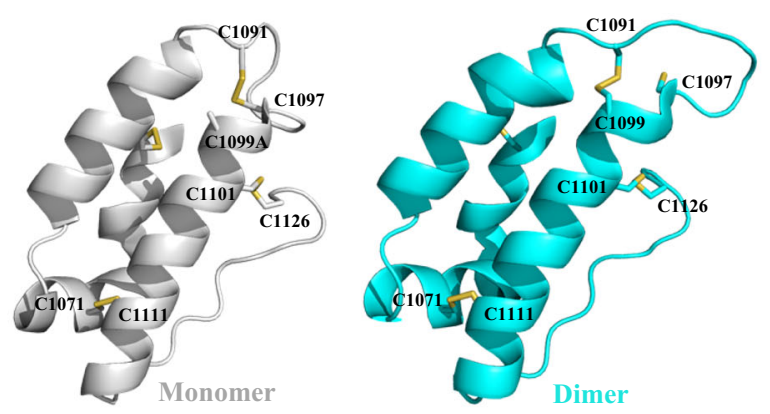

i

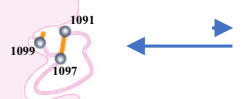

D'D3

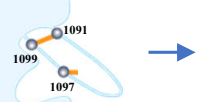

D’D3
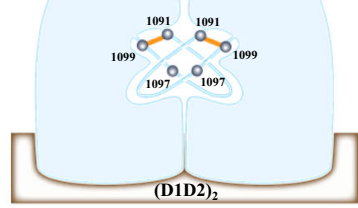

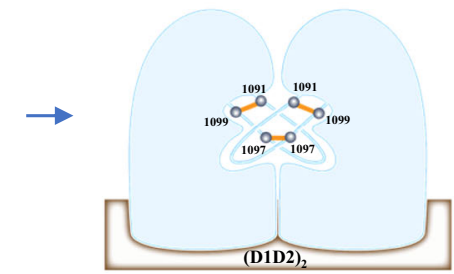

Fig. 1 (See legend on next page.) 
(see figure on previous page)

Fig. 1 Structural mechanism of $D^{\prime} D 3$ dimer formation. a The domain arrangements of full-length WWF. $\mathbf{b}$ Identification of disulfide-linked peptides from the $\mathrm{D}^{\prime} \mathrm{D} 3$ dimer interface. The $\mathrm{D}^{\prime} \mathrm{D} 3$ dimers were cleaved by $\mathrm{CNBr}$ and analyzed by mass spectrometry with Cys ${ }^{1097}$-Cys ${ }^{1097^{\prime}}$ peptides confirmed by the MS/MS spectrum. c Identification of free cysteines in monomeric D'D3. D'D3 monomers were sequentially modified by NEM and 4VP, then treated with protease Asp-N and analyzed by LC-MS. $\mathbf{d}$ Cryo-EM structure of the repeating unit containing one VWF D'D3 dimer (cyan and pink) and one D1D2 dimer (wheat), which mirrors the repeating unit of MUC2. Other intermolecular interfaces in the complex are shown in Supplementary Fig. S8. e The dimer shown in the cartoon with the WWD3 module of D3 (left chain) colored in brown, C8-3 in cyan, TIL3 in purple, and E3 in yellow and the other D3 chain in gray. $\mathbf{f}$ The electron densities covering the key disulfide bonds Cys ${ }^{1097}$-Cys ${ }^{1097^{\prime}}$ and Cys ${ }^{1091}$-Cys ${ }^{1099}$ near the D'D3 dimeric interface are shown as gray mesh contoured at $1.5 \mathrm{rmsd}$. $\mathbf{g}$ The overlaid structures of D'D3 monomer (gray) and dimer (cyan) showing the loop linking residues 1091 and 1099 flips up and stacks with the same loop from the other molecule in the dimer (Supplementary Fig. S5). h Relative positions of cysteines Cys ${ }^{1091}$, Cys ${ }^{1097}$, and Cys ${ }^{1099}$ in $D^{\prime} D 3$ monomer and dimer. i A diagram showing D'D3 monomer equilibrating in configurations with either Cys ${ }^{1097}$ or Cys ${ }^{1099}$ free. Only the main form with free Cys ${ }^{1099}$ was identified in the previous study possibly due to the lower sensitivity of the equipment or the lower amount of protein used. In the presence of WWF D1D2 domains at mild acidic pH, only D'D3 monomers with Cys $^{1091}-\mathrm{Cys}^{1099}$ bond could readily bind each other complimentarily and allow intermolecular Cys ${ }^{1097}$-Cys ${ }^{1097^{\prime}}$ disulfide bond formation.

unequivocally demonstrated the presence of Cys $^{1097}-$ Cys $^{1097^{\prime}}$ and Cys ${ }^{1142}-$ Cys $^{1142^{\prime}}$ linkages in the VWF D'D3 dimers.

We then tried to verify the free cysteines in the $\mathrm{D}^{\prime} \mathrm{D} 3$ monomer using the same differential alkylation procedure reported previously where $\mathrm{D}^{\prime} \mathrm{D} 3$ monomers were treated with N-ethylmaleimide (NEM) to block free thiols and the intramolecular disulfide bonds were reduced with dithiothreitol (DTT) and modified by 4-vinylpyridine (4$\mathrm{VP})^{5}$. The peptides $\left({ }^{1137}\right.$ ENGYEC(NEM)EWR $\left.{ }^{1145}\right)$ with Cys ${ }^{1142}$ modified by NEM could be readily detected from the tryptic digestion (data not shown). Interestingly, four peptide peaks were identified from the Asp-N digestion by liquid chromatography with identical doubly charged ions with $m / z=498.675$, which corresponded to peptide ${ }^{1096}$ DCACFC $^{1101}$ modified by one NEM and two 4-VP molecules (Fig. 1c). The peptides in the two major peaks (I and IV) were confirmed by the MS/MS spectrum to be ${ }^{1096} \mathrm{DC}(4-\mathrm{VP}) \mathrm{AC}(\mathrm{NEM}) \mathrm{FC}(4-\mathrm{VP}){ }^{1101}$ with two peaks representing two diastereomers from the NEM derivative. This is consistent with previous findings that Cys ${ }^{1099}$ in $\mathrm{D}^{\prime}$ D3 monomer could be modified by $\mathrm{NEM}^{5}$. The peptides in the two minor peaks (II, III) were identified to be ${ }^{1096} \mathrm{DC}(\mathrm{NEM}) \mathrm{AC}(4-\mathrm{VP}) \mathrm{FC}(4-\mathrm{VP}){ }^{1101}$, corresponding to those with Cys ${ }^{1097}$ modified by NEM (Fig. 1c). These results indicate that $\mathrm{Cys}^{1099}$ is the free cysteine in most $\mathrm{D}^{\prime}$ D3 monomers, but Cys ${ }^{1097}$ is free in a small amount of $D^{\prime}$ D3 monomers. Thus, it is highly plausible that there is an equilibrium of Cys ${ }^{1099}$ and Cys ${ }^{1097}$ forming an alternative disulfide bond with the same cysteine, most likely Cys ${ }^{1091}$, which may be consistent with the disulfide exchange mechanism proposed by Dong and Springer ${ }^{6,7}$. Notably, the replacement of either Cys ${ }^{1097}$ or Cys ${ }^{1142}$ alone would not prevent D'D3 dimerization, consistent with a similar mutagenesis study of $\mathrm{MUC}^{8}$ (Supplementary Fig. S2).

In order to gain further structural information of the D'D3 dimer interface, we purified D'D3 dimers complexed with D1D2 derived from the expression medium of HEK293 cells transfected with expression plasmid of D1D2D'D3-wt or D1D2D'D3-R1136M-E1143M.
The tubule-like oligomers of these complexes were analyzed by single-particle cryo-EM (Supplementary Figs. S3, S4). This yielded an electron density map covering two repeating units for D'D3-R1136ME1143M dimer complexed with D1D2 at $3.3 \AA$ resolution and an electron density map covering one repeating unit for D'D3-wt dimer complexed with D1D2 at $3.4 \AA$ resolution respectively (Supplementary Table S1). Each repeating unit contains a $\mathrm{D}^{\prime} \mathrm{D} 3$ dimer and a D1D2 dimer. As the overall configuration of the refined $D^{\prime} D 3-$ wt complex is essentially the same as that of the D'D3R1136M-E1143M mutant, the structure of the mutant was selected for presentation due to its slightly better resolution. The shape of the repeating unit largely resembles that of MUC2 where a D'D3 dimer is docked in the central hole of the donut-shaped D1D2 dimer ${ }^{8}$ (Fig. 1d, e). Since the crystal structure of a monomeric $\mathrm{D}^{\prime} \mathrm{D} 3$ variant has been solved previously ${ }^{6}$, this allowed us to build the $\mathrm{D}^{\prime} \mathrm{D} 3$ dimer structure with confidence (Fig. 1e; Supplementary Table S1). The electron density near the center covering the $\mathrm{D}^{\prime} \mathrm{D} 3$ dimer is unambiguous and a clear density covering the disulfide bonds near the dimeric interface can be observed (Fig. 1f).

The structure (Fig. 1e) showed that $\mathrm{D}^{\prime} \mathrm{D} 3$ retained the overall conformation seen in the crystal structure of $\mathrm{D}^{\prime} \mathrm{D} 3$ monomer (rmsd of $1.7 \AA$ ) but with local conformational rearrangements near the dimeric interface (Fig. $1 \mathrm{~g}$, $\mathrm{h}$ and Supplementary Figs. S5, S6). The connecting loop linking two helices in the $\mathrm{D}^{\prime} \mathrm{D} 3$ monomer restrained by the Cys $^{1091}$-Cys ${ }^{1097}$ disulfide is anchored in a hydrophobic pocket formed by $\mathrm{Phe}^{1100}$, Met $^{1055}$, and $\mathrm{Val}^{1056}$ through Ile ${ }^{1094}$, but in the dimer, it is more flexible with the intramolecular Cys ${ }^{1091}-\mathrm{Cys}^{1099}$ bond and flips over to dock in the same hydrophobic surface pocket of the other D'D3 molecule in a domain swapping-like fashion (Supplementary Fig. S6). This brings two Cys ${ }^{1097}$ residues in proximity and the subsequent formation of an intermolecular disulfide bond. As Cys ${ }^{1142}$ in a double-stranded hairpin was shielded from solvent exposure in the $\mathrm{D}^{\prime} \mathrm{D} 3$ monomer structure ${ }^{6}$, but rotated and shifted $\sim 6 \AA$ to form 
an intermolecular disulfide bond with its counterpart in the other D'D3 molecule in the dimer (Supplementary Figs. S5, S6), it seems that VWF has adopted an allosteric mechanism for the intermolecular disulfide formation with the free thiols in the monomers protected from surface exposure avoiding unwanted oxidation during the biosynthesis.

Overall, our studies here indicate that $\mathrm{D}^{\prime} \mathrm{D} 3$ monomer equilibriums between two configurations where Cys ${ }^{1091}$ forms an intramolecular disulfide bond with either $\mathrm{Cys}^{1097}$ or Cys ${ }^{1099}$ (Fig. 1i). However, only the minor D'D3 confirmation containing a longer connecting loop with the $\mathrm{Cys}^{1091}-\mathrm{Cys}^{1099}$ bond and free Cys ${ }^{1097}$ could allow domain swapping-like complementary binding of the two loops in the dimer interface and the formation of intermolecular Cys ${ }^{1097}$-Cys ${ }^{1097}$ disulfide bond in the presence of VWF domain D1D2s (Fig. 1i). As there are very limited noncovalent interactions in the $\mathrm{D}^{\prime} \mathrm{D} 3$ dimeric interface (Supplementary Fig. S6), D1D2 is indispensable for its dimerization by aligning two D3 monomers in optimal positions for their intermolecular disulfide formation (Fig. 1d, i). Although it remains unclear how the disulfides in the $\mathrm{D}^{\prime} \mathrm{D} 3$ dimer interface would reshuffle or form at $\sim \mathrm{pH} 5.8$ in Golgi and various mechanisms have been proposed $^{7,9}$, our cryo-EM structure of a D'D3 dimer complexed with D1D2s shows that the two Cys ${ }^{1097}$ are largely buried in the center of the dimeric interface and there is limited space to allow the participation of any bulky redox-regulating enzymes (Supplementary Figs. S7, S8). Therefore, it is plausible that only small redox molecules are directly involved in this process, which would be consistent with the previously observed formation of VWF oligomers in vitro ${ }^{10}$.

\section{Acknowledgements}

This work was supported in part by grants from the National Natural Science Foundation of China (32070934, 32171190 and 81870309) and Ministry of Science and Technology of China (2016YFA0501 103). We thank the Shanghai Science and Technology Commission (20JC1410100) and the Core Facilities of Basic Medical Sciences, Shanghai Jiaotong University School of Medicine for support. We thank the Tsinghua University Branch of China National Center for Protein Sciences (Beijing) for providing the cryo-EM facility support and Jianlin Lei, Xiaomin Li, and Fan Yang for technical support, and the support of computational facility on the cluster of Bio-Computing Platform. We thank Professor Jiawei Wang from

Tsinghua University for kind help and the cryo-EM access.

\section{Author details}

'Department of Pathophysiology, Key Laboratory of Cell Differentiation and Apoptosis of Chinese Ministry of Education, Shanghai Jiao Tong University School of Medicine, Shanghai, China. ${ }^{2}$ State Key Laboratory of Membrane Biology, Beijing Advanced Innovation Center for Structural Biology, School of Life Sciences, Tsinghua University, Beijing, China. ${ }^{3}$ Present address: Department of Biochemistry and Molecular Biophysics, Washington University in St. Louis, School of Medicine, St. Louis, MO, USA

\section{Author contributions}

A.Z. conceived the project. Z.S., J.Z., L.X., and H.C. performed the experiments. All authors analyzed the data and contributed to the paper preparation. A.Z. and Z.S. wrote the paper.

\section{Conflict of interest}

The authors declare no competing interests.

\section{Publisher's note}

Springer Nature remains neutral with regard to jurisdictional claims in published maps and institutional affiliations.

Supplementary information The online version contains supplementary material available at https://doi.org/10.1038/s41421-022-00378-2.

Received: 5 November 2021 Accepted: 20 January 2022

Published online: 15 February 2022

\section{References}

1. Huang, R. H. et al. Assembly of Weibel-Palade body-like tubules from $\mathrm{N}$-terminal domains of von Willebrand factor. Proc. Natl Acad. Sci. USA 105, 482-487 (2008)

2. Springer, T. A. von Willebrand factor, Jedi knight of the bloodstream. Blood 124, 1412-1425 (2014).

3. Lenting, P. J., Christophe, O. D. \& Denis, C. V. von Willebrand factor biosynthesis, secretion, and clearance: connecting the far ends. Blood 125, 2019-2028 (2015)

4. Dong, Z. et al. Disulfide bonds required to assemble functional von Willebrand factor multimers. J. Biol. Chem. 269, 6753-6758 (1994).

5. Purvis, A. R. et al. Two Cys residues essential for von Willebrand factor multimer assembly in the Golgi. Proc. Natl Acad. Sci. USA 104, 15647-15652 (2007).

6. Dong, $X$. et al. The von Willebrand factor $D^{\prime} D 3$ assembly and structural principles for factor VIII binding and concatemer biogenesis. Blood 133, 1523-1533 (2019)

7. Dong, X. \& Springer, T. A. Disulfide exchange in multimerization of von Willebrand factor and gel-forming mucins. Blood 137, 1263-1267 (2021).

8. Javitt, G. et al. Assembly mechanism of mucin and von Willebrand factor polymers. Cell 183, 717-729.e16 (2020).

9. Horowitz, B. et al. Quiescin sulfhydryl oxidase 1 (QSOX1) glycosite mutation perturbs secretion but not Golgi localization. Glycobiology 28, 580-591 (2018).

10. Mayadas, T. N. \& Wagner, D. D. In vitro multimerization of von Willebrand factor is triggered by low $\mathrm{pH}$. Importance of the propolypeptide and free sulfhydryls. J. Biol. Chem. 264, 13497-13503 (1989). 